\title{
Negative Knowledge: Understanding Professional Learning and Expertise
}

\author{
Martin Gartmeier • Johannes Bauer • \\ Hans Gruber • Helmut Heid
}

Received: 29 October 2007 / Accepted: 12 February 2008 /

Published online: 26 February 2008

(C) Springer Science + Business Media B.V. 2008

\begin{abstract}
In this paper, we critically analyze how the concept of negative knowledge contributes to the understanding of professionals' expert practice and learning. Negative knowledge is experientially acquired knowledge about what is wrong and what is to be avoided during performance in a given work situation. In terms of its theoretical foundation, the concept relates to constructivist theorization and metacognition. Building on existing conceptions of negative knowledge, we systematically relate the concept to research on expertise and learning from errors. The concept of negative knowledge augments existing theories of professional knowledge by emphasizing knowing about what to avoid as part of experts' effective actions. During routine actions, negative knowledge enhances professionals' certainty of how to proceed and increases the efficacy through the avoidance of impasses and suboptimal problem-solving strategies. Quality and depth of reflective processes after actions are related to the development of negative knowledge. The potential of negative knowledge for the investigation of professional learning is discussed through reference to recent empirical work.
\end{abstract}

Keywords Negative knowledge · Learning from errors · Professional learning · Workplace learning

Avoiding serious errors is an important quality of professional expertise. One explanation for expert performance is associated with the ability to avoid severe errors. A plausible, but yet not widely considered explanation for such a capacity is the availability of explicit knowledge about what not to do in certain situations - so called negative knowledge. Here, we propose that integrating the concept of negative knowledge into research on professional learning and on expertise is worthwhile.

Re-submitted for publication in: Vocations and Learning: Studies in Vocational and Professional Education.

M. Gartmeier $(\bowtie) \cdot$ J. Bauer $\cdot$ H. Gruber $\cdot$ H. Heid Institute of Educational Science, University of Regensburg, 93040 Regensburg, Germany e-mail: martin.gartmeier@paedagogik.uni-regensburg.de 
This is because negative knowledge engages with an important aspect of effective action that has so far not been sufficiently considered. An account of this concept adds to discussions in the field of workplace learning. As elaborated in this paper, negative knowledge has the potential to enrich the discussion around learning from errors at work through explaining how individuals' reflection of and learning through these experiences benefit in the long term. Furthermore, research on expertise has not yet appropriately addressed the question if and how experts and novices in a professional field differ in the ways they use negative knowledge. Moreover, we advance the position that negative knowledge is of general interest to the field of professional work and learning, because it contributes to the expertise of professionals more generally.

Firstly, negative knowledge fosters certainty in professionals' action. Of course, it is crucial to know what actions to take when solving a problem. However, since in most cases there are several possibilities to mistake making, an awareness of what approaches are suboptimal or what actions are to avoid during a problem solving process should assist individuals' notion of certainty in their professional practice. Secondly, negative knowledge directly influences performance by allowing professionals to identify and correct inadequate ways to proceed and thus increases their efficiency of problem solving. Thirdly, negative knowledge has an important heuristic value and promotes the quality and depth of reflection on action.

In making this case, our argument commences with discussing the potential of negative knowledge through theoretical foundations of constructivism and metacognition. Then, conceptions of negative knowledge are discussed as developed in the fields of education and business philosophy (Minsky 1994; Oser and Spychiger 2005; Parviainen and Eriksson 2006). However, our goal is to go beyond existing contributions by systematically relating the concept of negative knowledge to research on professional expertise (Ericsson et al. 2006) and workplace learning (Gruber and Palonen 2007) to appraise its potential for effective professional practice. These theoretical considerations are then integrated in framing empirical investigation of the development, structure and function of negative knowledge.

\section{Conceptions of Negative Knowledge}

In this section, the concept of negative knowledge and its theoretical foundations are discussed. For highlighting the theoretical reference points of the terms "negative" and "knowledge", relevant aspects of constructivist theorization as well as metacognition are used to describe this concept.

\section{Theoretical Foundations}

To establish a theoretical framework, it is necessary to define what characterizes the term "negative" in negative knowledge. A common misunderstanding to be addressed here is that "negative" knowledge is bad, disadvantageous, or malign. Just like negative numbers not being either good or bad, labeling knowledge as being "negative" does not imply a valuation. Instead, the term "negative" refers to characteristic attributes described below. To clarify an important attribute of negative 
knowledge requires considering the use of the term "knowledge" in constructivist theorization. In constructivism, knowledge is not seen as an exact representation of reality, but rather as a

map of what reality allows us to do. It is the repertoire of concepts, conceptual relations, and actions or operations that have proven to be viable in the pursuit of our goals. (von Glasersfeld 1991, p. 3, original emphasis).

In that sense, knowledge in a constructivist understanding is regarded as a system of representations and assumptions about reality that is closely related to individual goals and their achievement. Roughly speaking, this means that individuals' assumptions can be called viable if they do not (severely) contradict previous knowledge and turn out to be useful for reaching actual goals. Yet again, non-viable knowledge is knowledge that somehow stands in contradiction to prior knowledge or is counterproductive with regard to a certain goal.

The basic idea that is pursued through the concept of negative knowledge is that just because knowledge is non-viable in the described understandings, it is not necessary worthless or superfluous. This is because in order to reach a goal, there are often different ways that seem possible and the task of identifying the right one is very complex and demanding. Therefore, it is seen as having a heuristic advantage through knowing what is wrong in regard to a certain task: that is, to have negative knowledge. Hence, negative knowledge can be described as non-viable knowledge that is heuristically valuable.

In the above given quote of von Glasersfeld (1991), knowledge is compared to a map. Remaining with that metaphor, negative knowledge can be seen as an indicator of adverse ways, wrong turns or disadvantageous routes in order to reach a certain destination. An example: A taxi driver in New York who knows that, despite it being the shortest way, taking Broadway when going from Central Park West to Manhattan Bridge is a mistake, maybe because there are severe traffic congestions because of ongoing construction work. This knowledge offers the advantage of securing the goal of quickly bringing his fares to their destination. His negative knowledge "Do not drive via Broadway!" enables him to avoid an unfavorable decision and to optimize his performance by choosing a quicker route. In this example, the taxidrivers negative knowledge has procedural character. It relates to an action that is not helpful or even counterproductive with regard to the intended goal. Yet, the "nonviability" of negative knowledge can also relate to facts. Non-viable facts are untrue with regard to an accepted criterion. This distinction presupposes the two main facets of negative knowledge that will be elaborated later in this paper.

Before this happens, the theoretical concept of knowledge pursued in the investigation of negative knowledge needs to be clarified. Briefly said, negative knowledge is to be regarded as metacognitive knowledge (Flavell 1979, Smith 2003). Metacognitive knowledge involves "knowledge of general strategies that might be used for different tasks, knowledge of the conditions under which these strategies might be used, knowledge of the extent to which the strategies are effective, and knowledge of self" (Pintrich 2002, p. 219). This definition holds metacognitive knowledge as having an important regulative function enabling individuals to adapt action strategies to the conditions of their application. Furthermore, it involves awareness of one's own competencies and knowledge and stands as an 
important prerequisite of competent action and professional development (Eraut 1994). Two plausible ways in which metacognitive knowledge regulates actual behaviour are to amplify or trigger appropriate or to inhibit inappropriate action strategies. In containing information about false facts and inappropriate actions or strategies, the regulative function of negative knowledge is expressed through an inhibitive effect. This again means that to apply negative knowledge, in terms of perceivable behavior, primarily means not to do something or to interrupt a course of action. This is consistent with the above described taxi-example: To apply negative knowledge means to avoid a suboptimal route. This effect of metacognitive regulation will be further illustrated through accounts of the procedural and declarative aspect of negative knowledge.

\section{Procedural and Declarative Negative Knowledge}

Building on the presented theoretical foundations, this section seeks to define negative knowledge and explore its procedural and declarative aspect. Along with the characterization of the two facets, their value for understanding professional learning and performance is exemplified.

On the one hand, negative knowledge is understood as part of the practical knowhow of professionals relating to tasks and activities specific for their workplace or professional domain (Minsky 1994; Oser and Spychiger 2005). This aspect can be paraphrased as "to know what not to do". In the context of this paper, it is addressed as the procedural aspect of negative knowledge. On the other hand, a different focus emerged, e.g. through Parviainen and Eriksson (2006) describing knowledge about deficient aspects of one's own knowledge as negative knowledge. This notion is different from the procedural aspect, as it is not focused on actions or strategies. It is better described as "to know what not to know" and shall hence be referred to as the declarative aspect of negative knowledge. Our examination turns to the procedural aspect first.

An early conception of negative knowledge is found within Minsky's (1994) conception of negative expertise. He argues that experts have a substantial amount of knowledge about what can go wrong in their domain and what kind of procedures will lead to suboptimal solutions of problems and, therefore, have to be avoided. Minsky (1994) concludes that this knowledge supports experts' effective action and prevents them from committing errors. However, he did not elaborate further or conduct empirical research on his concept of negative knowledge.

A similar concept with a focus on educational settings was advanced by Oser and Spychiger (2005). They define negative knowledge as knowledge about "what something is not, (in contrast to what it is), and how something does not work, (in contrast to how it works), which strategies do not lead to the solution of complex problems (in contrast to those, that do so) and why certain connections do not add up (in contrast to why they add up)" (Oser and Spychiger 2005, p. 26, translated by the authors). This definition bolsters the assumption that negative knowledge has to be regarded as a form of meta-knowledge containing information about false facts and inappropriate action strategies. An interesting aspect in this definition is the emphasis on the contrastive character of negative knowledge. This includes its regulative function as described above. Knowing what not to do in a situation surely 
does not suffice in order to act right. Yet again, during identifying a promising strategy for solving a problem, one might profit from knowing about strategies that have turned out to be bad or suboptimal in the past and from modifying the new strategy according to the "lessons learned" during previous experience. To illustrate this understanding of negative knowledge, Oser and Spychiger (2005) provide a workplace-related example:

In a Nepalese hospital, a local doctor introduces visiting physicians by confronting them with "Seven easy ways to kill a newborn infant (without even trying)", for example "Do not monitor vital signs", "Feed infants with buffalo milk", "Keep newborn infants with other patients who have contagious illnesses or draining wounds" or "Do not wash your hands prior to examining infants" (Oser and Spychiger 2005)

This episode reveals characteristics of negative knowledge, with the most prominent being its primary origin in individual experience. The list of ways to kill a newborn certainly does not represent pieces of medical textbook knowledge. Instead, it arises from practical experience within concrete work contexts, in which physicians are confronted with a particular set of complexities and constraints demanding the adherence to specialized knowledge obtained through others' or their own experience. The position advanced in this paper is that the experiential character of negative knowledge is an important theoretical link to the contexts of professional learning and expertise.

Contrasting the described procedural aspect, a recent perspective stressed out the value of a declarative conception of negative knowledge being focused on deficient aspects of one's own (professional) knowledge (Parviainen and Eriksson 2006). Within such a perspective negative knowledge could be paraphrased as "to know what not to know" rather than "to know what not to do". To illustrate this conception of negative knowledge, the authors claim that "we seek knowledge because we do not know something and we are aware of this ignorance" (Parviainen and Eriksson 2006, p. 143). Here, the authors denote two different degrees of not-knowing. On the one hand, an uninformed way of not-knowing implies an individual not knowing something that would be crucial for him and, moreover, not being aware of this lack of relevant knowledge. On the other hand, an informed way of not knowing describes an individual being aware of one's own lack of relevant knowledge. This conception relates to a dualistic distinction on the level of competence as introduced by Heid (1996). Conceiving competence as the ability to solve problems, Heid (1996) distinguishes between trivial and non-trivial competence. A case of trivial competence occurs when individuals succeed in solving a task without really knowing why. In contrast, non-trivial competence means to be able to repeatedly bring about the success, because one knows about the reasons and conditions under which it is achieved. The idea of negative knowledge goes one step further here in amending these categories by a notion of trivial and non-trivial incompetence. The latter means that individuals know what they are not competent for, which can have different advantageous consequences. For instance, they might set out to deliberately learn the new skill or call on another person who is known to them for having the relevant knowledge or competence. The conception of Heid (1996) is consistent with Parviainen and Eriksson's (2006) idea. That is, regardless if one does not know 
something that he should know or is not able to perform a task properly - a crucial prerequisite of the learning processes that seem necessary in both cases is awareness about the absence of relevant skills or information in the form of negative knowledge.

The importance of this understanding of negative knowledge becomes obvious when the dynamic nature of many current workplaces is considered. Profound changes bring about situations where relevant and applicable knowledge becomes obsolete and is turned into non-viable or expired knowledge. In such situations, an individual awareness about knowledge that is to be reconsidered or updated is a crucial factor in developing and maintaining professional competence. The more precisely individuals manage to detect lacks of relevant know-how as well as of outdated knowledge being still "in use", the better it should be possible to recognize what is to be learned. According to Parviainen and Eriksson (2006), the ability of "unlearning and bracketing knowledge" (p. 144) is closely connected to an explicit individual knowledge of lacks, shortcomings or deficits in one's own knowledge base. This meta-cognitive notion of negative knowledge is a perspective relating to current discussions on expertise, which Parviainen and Eriksson (2006, p. 151) label as a "reflective turn" in this field. It follows then that this features of expertise, like doubt and awareness of own errors and weaknesses, are important additions to traditional conceptions of expertise.

The procedural and the declarative perspectives on negative knowledge discussed here are complementary. They represent two different ways to apply the idea of negativity within the general domain of individual knowledge. We seek to advance both understandings to elaborate the concept of negative knowledge in its multiple understandings and relating it to research on professional learning and expertise. Thus, the points made in the following sections are inclusive of both conceptions.

\section{Functions of Negative Knowledge}

Drawing on what has been advanced so far, we propose that negative knowledge has a number of beneficial effects in professional contexts, because it: (1) supports certainty in how to proceed; (2) increases efficiency during actions; and (3) enhances the quality and depth of reflection processes on action. These assertions are now discussed in turn.

\section{Negative Knowledge Increases Certainty}

It is assumed that negative knowledge helps to increase individuals' certainty through awareness of possible positive as well as negative outcomes of their actions and through the capability to judge their respective probabilities under given circumstances. As advanced by a number of scholars (e.g. Frese 1991, 1995; Perrow 1984; Reason 1990; Rochlin 1999), it seems impossible to completely prevent human error in work life, because complex human action such as is conducted by professionals is prone to errors. This raises the question how individuals, faced with a constant risk of going wrong in their actions and decisions, can ever realise certainty and self-assuredness in their professional practice. Existing concepts that 
proved to have explanative power here are e.g. self-efficacy (Bandura 1997) tolerance of ambiguity (Furnham and Ribchester 1995) or counterfactual thinking (Morris and Moore 2000). Yet, we propose that a notion of certainty in work situations can also be explained by means of an individuals' explicit and precise knowledge about what things might go wrong and, in turn, about what actions to avoid in a specific situation.

\section{Negative Knowledge Increases Efficiency}

Negative knowledge is assumed to contribute to effective action. There is already some evidence for this conjecture from research on the organizational level. Van Dyck et al. (2005) show an impact of organizational error management culturewhich should foster the creation of negative knowledge among employees - on company performance. Error management culture was operationalized in a number of facets like communication about errors, coordination of error handling, and sharing error knowledge. These variables were positively related with a number of measures of company performance (i.e. goal achievement, survivability and return on assets). The results, which are consonant with those of a number of other studies (Edmondson 1996; Helmreich and Merritt 2000; Rochlin 1999), support the assumption that the availability of negative knowledge increases efficiency on an organizational level. Although these conclusions cannot be generalized to the individual level, they encourage further investigations about what effects transfer.

\section{Negative Knowledge Promotes Reflection}

Negative knowledge is assumed to promote detailed reflective processes, because an essential component of reflection comprises an engagement with individual's prior and episodic knowledge. Reflection is commonly conceptualized as a process of reexamination and re-appraisal of encountered experience. Its importance for the development of professional competence was emphasized in various recent studies (Boud 1999; Ellström 2006; van Woerkom 2003). Drawing on this general role of knowledge as an element of reflective processes, we propose that negative knowledge is a stimulating element within reflective processes due to its heuristic function. Remembering and being aware what is inappropriate in a given situation should enhance the ability to precisely discriminate similar phenomena. Consequently, it stimulates the analysis of encountered problems from different perspectives in subsequent analyses of the respective episode (Oser and Spychiger 2005).

In sum, the concept of negative knowledge contributes to the understanding of expert performance by elaborating a broader base of professionals' knowledge and performance. Therefore, in order to elaborate these issues, the concept of negative knowledge, needs to be related to existing research on professional development and expertise.

\section{Negative Knowledge and Existing Theorization-Exploring Its Compatibility}

Before the potential of the negative knowledge concept is addressed, clarification is necessary in what ways it relates to what we already know about professional 
learning and development. First, it is proposed that the theoretical structure of the concept of negative knowledge and, secondly, its theoretical derivates are consistent with models of dynamic changes in expert memory through learning from critical experience.

\section{Negative Knowledge and Critical Incidents in Professional Experience}

Research on professional learning and development is strongly based on studies and conceptualizations of experiential learning (Boshuizen et al. 2004). Through this form of learning, the construction of new knowledge and the modification of existing knowledge are achieved through the active participation in personally meaningful episodes in an authentic context (e.g. a workplace). Critical questions here are how experiential learning takes place and how it influences later performance. We turn to the latter here and discuss the former in the following section.

The concept of dynamic memory shows how knowledge structures (i.e. scripts) change according to experiences of deviance (e.g. after occurrence of an error) if the episode is analyzed through reflection. These knowledge structures assist performance in later similar situations by recalling the deviant episode during case-based reasoning (Kolodner 1993). The focus on aspects of critical episodes which are perceived as "wrong, but relevant" enriches experiential learning processes, because negative knowledge matches or challenges assumptions concerning relevant memory structures. Thus, the beneficial effects of negative knowledge are closely related to assumptions from conceptions of experiential learning.

An example how negative knowledge drawn from critical experience fosters competence development is found in the vocational education of airplane pilots. A crucial element of their job training takes place within the artificial environment of a flight simulator. The advantage of a flight simulator as a learning environment is that it enables trainees to learn from (almost) authentic critical experience without facing the fatal consequences that wrong actions would have, were they carried out in a real airplane (Wood and Sweggins 2006). One valuable result of such training in the safe environment of a simulator (besides staying alive) can be described as procedural negative knowledge about what actions or conditions are to be avoided in all circumstances when flying an airplane.

The collection of experience, especially critical experience, very often is a thoroughly emotional process. Therefore, another aspect of experiential knowledge is its strong emotional character (Eraut 1994). According to existing conceptions, this seems particularly true for negative knowledge. As a "mental immune system" (Oser and Spychiger 2005, p. 42), negative knowledge can prevent an individual from repeating errors which were experienced earlier in a similar manner. This aspect will be explicitly addressed in the following sections.

\section{Learning from Errors}

Learning from errors is important for the development of negative knowledge (Oser and Spychiger 2005). It is difficult to define what constitutes an error in general, because errors involve an evaluation based on norms, the interpretation and acceptance of which may differ substantially between workplaces or even between 黑 Springer 
individuals at one workplace (Harteis et al. 2007). The position taken here is that individual errors can be seen as actions that endanger the attainment of desired goals. Furthermore, labeling an action as an error involves the judgment of skilful members of the community (Heid 1999; Reason 1990).

If we assume negative knowledge to be the outcome of learning from errors, the question is how learning from errors can be conceptualized and why it should lead to knowledge that has the assumed features of negative knowledge. Learning from errors at work can be conceptualized as a special form of experiential learning and thus plays a crucial role in professional development. To revisit the given example: For pilots, the consequences of certain errors are so severe that it makes sense to create an extraordinarily complex simulated training environment. For many other professions, such simulated environments do not exist. Therefore, learning at the workplace mostly means learning from real, sometimes painful experience (van Woerkom 2003).

As a special case of experiential learning, error-related learning employs the construction of knowledge from episodic events. Errors have the potential to challenge individual mental models and thus provide starting points for modification of these models (Ellis and Davidi 2005; Frese and Zapf 1994; Kolodner 1983; Schank 1999). Such processes of modification can happen in different modes, e.g. through differentiation of the underlying model of action, through replacement of parts of the model, or through indexing false connections or weak areas within the model that bring about potentials for errors. We assume these processes to be the building blocks for the construction of negative knowledge.

A related concern, it follows, is which learning activities mediate between the experience and the revision of knowledge. It is plausible that analysis and reflection are crucial processes, especially in situations when it is not very obvious what kind of error has occurred and what would be the optimal way to correct it. Furthermore, Oser and Spychiger (2005) propose that also regret and correction are required for building negative knowledge from an error. On the basis of the experiential learning theory by Kolb (1984), Bauer and Mulder (2007) suggest a framework of errorrelated learning activities, involving: (1) cause analysis, (2) the development of new strategies in order to avoid the error in future, and (3) experimentation with and implementation of these new strategies to the work context. This framework can be interpreted in terms of the creation, application, and further modification of knowledge that is focused on what is wrong, but relevant.

In sum, the concept of negative knowledge can be plausibly related to existing theories in the context of professional learning. In the next section, we argue that it has the epistemic potential to develop new insights which justifies its introduction as a new concept.

\section{Added-value of a Knowledge Perspective for Research on Professional Learning and Expertise}

As proposed, the concept of negative knowledge and its efficacy is strongly connected to existing research on professional development and expertise. Yet, it has the potential to significantly enrich these theoretical contexts. Firstly, it avoids 
shortcomings of some current approaches to learning from errors by stressing the episodic character of experience and thus offers a perspective to analyze long term benefits of learning from errors on the cognitive level. Secondly, it contributes to the understanding of the nature of expertise. These perspectives will be elaborated in the following sections.

\section{Added-value of a Knowledge Perspective for Research on Error-related Learning Processes}

The concept of negative knowledge enriches the discussion about learning from errors by overcoming some existing shortcomings of current approaches. These shortcomings are especially due to the limitations of questionnaire methods for researching learning from errors as a subject with strong episodic quality. To substantiate the added value of a knowledge-based approach in this context, one current and widely used instrument in this area, the Error Orientation Questionnaire (EOQ; Rybowiak et al. 1999 ) is helpful. In this questionnaire, three scales addressing different aspects of coping with and thinking about errors at the workplace are error competence (item example: "When I have made a error, I know immediately how to correct it"), learning from errors (item example: "Mistakes assist me to improve my work"), and error strain (item example: "I find it stressful when I err") are informative.

However, deliberate reasoning about the example-questions used can lead to serious methodological difficulties. Most individuals probably remember encountering errors that they could correct immediately; just as well, error-episodes may come to one's mind, which were most stressful and difficult to analyze and to resolve. Moreover, while certain errors may have been very informative for one's own practice, others, especially careless errors, were committed despite individuals knowing better. An underlying problem is the term "error" being a general label for different phenomena, which moreover can be very diversely perceived and interpreted due to individual characteristics. This notion is supported by a study conducted by Harteis et. al. (2007). The authors asked employees to remember error episodes from their workplaces and thereby revealed large interindividual differences in what was identified as an error as well as in how the events were interpreted.

Thus, without drawing the substantial contributions of questionnaires like the EOQ to the field into question, it appears that research on individual error-handling deals with such a diverse and multifaceted subject that a greater variety of approaches should be introduced to get a more differentiated understanding of the phenomenon. The concept of negative knowledge allows for the analysis of errorrelated learning processes and addresses the above-mentioned difficulties. An error is perceived and treated as a singular event which causes individuals to initiate a process of reconceptualization and reorganization of parts of their knowledge. Episodic markers as well as conclusions drawn from an episode are used in individual memory as mental warning signs that can guide further action and experience (Kolodner 1983, Oser and Spychiger 2005). Put plainly, we propose that research on individual handling of critical professional episodes might profit from a focus on mental representations related to distinct examples of such episodes. That means the initial focus of research is narrowed to errors that occur in the context of special work tasks, projects or even to singular error events recalled by subjects in 黑 Springer 
order to capture the complexity of singular error events and to explore the interrelations between experience and individual knowledge.

Another argument supporting this perspective is that the substantial complexity of much modern workplaces makes it impossible to totally prevent errors. Nevertheless, precursors of errors can be analyzed based on prior experience. Such analyses may help to predict errors and, thus, to be prepared not to repeat them. The idea of negative knowledge offers a very plausible explanation for this effect. That is, knowledge learned from an error episode facilitates the identification of similar subsequent episodes by paying attention to precursors. This error-prevention capacity cannot satisfactorily be explained through individual orientations, as concrete and applicable knowledge is an important prerequisite for judging competently in complex situations.

\section{Added-value of a Knowledge Perspective on Expertise}

A knowledge-based interpretation of learning from errors can easily be related to research on expertise. As noted, an extensive repertoire of experience-based knowledge is regarded as the most important attribute of experts (Ericsson et al. 2006). Theories of expertise development stress the role of cognitive restructuring of domain knowledge through deliberate practice and extended experience. In that sense, Schmidt and Boshuizen (1993) showed how the process of knowledge encapsulation contributes to medical experts' superior ability to identify and solve diagnostic problems. The reflective evaluation of experienced episodes is used to modify domain knowledge and to integrate constraints and affordances of the workplace and of typical and atypical cases which are related to routine cases ("Do it as always!") or to exceptional, frequently negatively defined cases ("Do not do it as always!"). The notion of negative knowledge contributes to this discussion, because it helps to understand how experts evaluate a given problem situation, such as those regularly encountered in professional work. An expert does not only (almost) always know what to do and is able to do it quickly and effectively - experts also know what not to do and thus have the ability to avoid errors and to intercept and interdict unproductive lines of thought during performance (Minsky 1994). This suggests that the development from novice to expert could not only be described as an increase of knowledge, skills or performance quality, but also as a decrease of ineffective actions and errors. Some interesting empirical results on the interplay between expertise and error-rate in computer work come from Brodbeck et al. (1992), who surprisingly did not find any significant differences between computer novices and experts concerning the total number of errors that were committed. Yet, a closer examination of the type of errors committed reveals that novices made far more errors that were due to a lack of domain specific knowledge. However, experts who could rely much more on routine procedures committed a larger amount of routine errors (e.g. when a routine was applied in a wrong situation or when some important signal or sign was not noticed).

Relating these results to the concept of negative knowledge, one could assume that maybe compared to novices, experts do not have to refer as intensively to the functions of negative knowledge, because it has become a component of their differentiated repertoire of routines. This means that negative knowledge would decrease in amount and relevance during expertise development, which again would 
contradict widely documented findings of experts exceeding novices in terms of their knowledge, regardless which kind of knowledge is considered. In the next section, a way of investigating this assumption will be discussed.

In sum, we propose that the concept of negative knowledge augments existing theories of workplace learning through opening up new perspectives on learning from errors and research on expertise. Yet, a concern about the concept might be that it advances a notion of workplace learning as an overly individualistic project and thereby neglects the growing importance of socio-cultural perspectives (Fenwick 2006). Such critique does not fully recognize the potential of the concept. At each workplace, processes of negotiation take place between individuals through which (explicit or implicit) criteria are developed that determine what is accepted as proficient and inadequate practice (Rochlin 1999). Conceptualizing knowledge as being "negative" means putting it in relation to these criteria as well as to the social processes in which they are established. Therefore, the concept is apt to serve as a conceptual basis for studies researching vocational contexts under a socio-cultural perspective. However, as a general strategy to start researching negative knowledge empirically, we have chosen to seek evidence on an individual, cognitive perspective before expanding the scope to a socio-cultural level (Morris and Moore 2000). Below, we elaborate this strategy by sketching current and planned research activities.

\section{Negative Knowledge and Professional Learning: Outlining a Research Agenda}

With the aim to inspire empirical research from the discussion above, we delineate some assumptions for empirical inquiry. Through that, insights into ongoing projects researching negative knowledge are given with three foci, the structure, development, and function of negative knowledge.

\section{Cognitive Structure and Measurement of Negative Knowledge}

In order to empirically investigate negative knowledge, qualitative techniques of knowledge elicitation (Hoffman and Lintern 2006) like the prompting task technique (PTT; Brewer 1986) and the critical incident technique (CIT; Flanagan 1954) stand as possible starting points.

The PTT is an experimental technique in which individuals are presented different keywords or small phrases that activate cognitive content related to the given prompt. Thereby, it is possible to specify cognitive structures, e.g. in analyses of the autobiographical memory. Crovitz and Schiffman (1974) requested subjects to recall specific events they had encountered and to refer to these in their declarations induced by different prompts. Inspired by this approach, the PTT is currently used to gain insight into workplace-related negative knowledge in a study conducted in the domain of aged care nursing. Subjects are confronted with 20 diagnoses of varying typicality for this professional context, like social isolation, impaired verbal communication or cardiac insufficiency. Each diagnosis is accompanied by two questions: "What do you think one should pay special attention to in interaction with elderly people with the following diagnosis?" and "What should be avoided?". The subjects are advised to voice every thought going through their mind in course of 
their pondering about the prompts and diagnoses. They are told that there are no wrong or right answers and that it is important to consider both questions in their answers. Concerning the formulation of the input-questions, informal interviews were conducted in advance to achieve an appropriate selection of input diagnoses. These interviews revealed that the question "What should be avoided?" sometimes was experienced as being quite difficult to answer. These initial difficulties showed to diminish if the interviewees conducted some general reflection on the demands induced by the specific diagnosis. Most of the subjects, then, were able to identify relevant negative knowledge. For this reason, the question directly aiming at the subjects' negative knowledge was combined with the more general and more intuitively answerable question to what one should pay special attention to.

As evident in this example, the PTT allows the consideration of a broad array of key issues for a professional context. Therefore, research questions to be addressed by means of prompting task-studies are: "What basic forms of negative knowledge appear in a professional context?" "Concluding from these forms, what functions does it serve for professionals?", and "What roles do the procedural and the declarative aspect of negative knowledge play?"

One critical point about the rather broad focus of the PTT is that it may induce difficulties for the subjects to dig up their negative knowledge in the first place. Thus, one possibly interesting way to improve the methodology for the purpose of assessing negative knowledge could be to modify the rather abstract nature of the prompts to have a stronger episodic character. As we assume negative knowledge to have a prominent episodic quality, this could lead to a more targeted activation of this special aspect of the subjects' knowledge.

However, a general limitation of the PTT is that it does not allow for the researcher to influence the situation beyond the presentation of the prompts. In cases in which the researcher would want the subjects to further elaborate on interesting aspects of their knowledge, the PTT does not permit any directive intervention during the data collection.

In that way, the PTT is a method that allows putting a research focus on negative knowledge in its breadth. A different, yet equally interesting approach could facilitate a stronger focus on negative knowledge in its depth: The critical incident technique (CIT). This technique allows for eliciting episodes of negative knowledge, for tracing their origins in past individual experience, and for an exploration of the complexity of present conditions under which they are to be attended.

The CIT is based on the assumption that critical incidents are events holding significant potential for gaining insight into error-prone aspects of a work context (Flanagan 1954). Yet, starting out from critical incidents could also promote the reconstruction of individuals' negative knowledge. Within the hitherto characterization of negative knowledge as experiential knowledge, one main focus has been set on error-related learning. As errors at the workplace are often described as being stressful events and having a critical character, it can be assumed that they are both, incidents which are sufficiently outstanding to vividly remain in individual memory and episodic reference points of individuals' negative knowledge.

In that, research questions that could be addressed by means of CIT studies are: "Which episodes or experiences is negative knowledge related to?" "What are characteristics of these experiences?" "How is the process reconstructed from 
encountering the described experiences to drawing abstract knowledge from them?" "Which factors influenced that process?" "Concerning the relevance of negative knowledge - is it seen as subjective or rather as of general relevance?" "If so, is it shared with colleagues?"

Regarding the conception of studies building upon these ideas, the CIT offers a general framework rather than concrete methodological guidelines. For the conception of actual studies, a two phase design seems advantageous. A plausible aim of the first phase is the identification of incidents or situations being experienced critical during working. For this purpose, a group of innovative methods that could be combined with the CIT follow the general idea of ambulatory assessment (Fahrenberg and Myrtek 1996). These methods (e.g. photo elicitation) seek to assess special phenomena very close to their occurrence in work practice. In the second phase of such studies, the identified critical incidents can serve as a basis for semistructured interviews aiming at an identification of the subjects' personal theories and knowledge that guided their action in the concrete cases as well as the origins of their knowledge in previous experience.

\section{Development and Function of Negative Knowledge}

Theoretical assumptions suggest combining investigations of the development of negative knowledge with the analysis of its functions as these may vary in different phases of professional development. One approach to gain evidence how negative knowledge evolves during professional development is the application of the above described techniques within longitudinal and quasi-longitudinal studies. In both cases, the goal is to open up quantitative (e.g., How much negative knowledge is uttered by individuals of different degrees of expertise?) as well as qualitative perspectives (e.g., In what forms does negative knowledge appear? What functions does it serve in different stages of expertise?). It is still an unresolved question if experts' superior availability of routines leads to a decreased importance of their explicitly available negative knowledge. In an on-going study, the PTT is applied in a quasi-longitudinal design comparing three groups of employees in banks, drawing on the assumption of ten years of necessary preparation for gaining expert level. Novices (i.e. those with less than two years of professional experience) are compared with semi-experts (up to ten years of professional experience) and experts (i.e. those with more than ten years of professional experience). The goal of the study is to assess negative knowledge of the subjects and to analyze its roles at different phases of professional life.

Another approach is to conduct longitudinal studies with a repeated measurement of negative knowledge with the same subjects. Such an approach is in particularly promising during phases of professional life when vocational experience is collected within a new or a radically changed work environment. In the sense of an initially peripheral (Billett 2001; Lave and Wenger 1991), yet center-oriented participation, it is plausible that in these cases the development of expertise depends upon a continued accumulation of workplace specific knowledge through a large number of micro-learning processes. These reflect how the new professional environment and its challenges are tackled. Because of the increased need to avoid and cope with own errors during that time, the functions of negative knowledge that were hypothesized, in particular certainty and reflexivity, seem to be crucial for the development of 
expertise. Concrete knowledge about what to avoid within all circumstances in a professional context may be evident and trivial for an expert. Yet, as illustrated in the earlier pediatrics example, a basic "negative framework" comprising such "don'ts" might be crucial as an orientation system for the professional guidance of a novice.

The introduced foci are just a few examples among a large variety of research perspectives and questions to be addressed by means of applying the negative knowledge concept in the context of workplace learning. Other approaches could, for example, tie in with ongoing sociocultural discussions and could ask to which degree negative knowledge is part of the shared knowledge of a work group or company. This again could be investigated in relation to the company's error-culture, to existing routines of error-communication or to processes and opportunities of collaborative reflection.

\section{Conclusion}

In our contribution, we have explored the explanative potential of the concept of negative knowledge for understanding professional development and expertise. The goal was to offer this concept as a new conceptual tool that allows analyses of professionals' knowledge, action, and learning. We propose that negative knowledge is an important part of professionals' knowledge serving important functions in work contexts, such as: (1) providing certainty, (2) improving efficient action, and (3) fostering reflexivity. From our analysis of conceptions of negative knowledge, we concluded that it can be well integrated in existing theoretical models of professional knowledge and learning. However, it has an added explanative potential that goes beyond these existing theories, therefore justifying its introduction as a new concept. This added value of the concept of negative knowledge lies in opening up new perspectives within research in learning from errors and expertise. Finally, we outline a research agenda of current or planned studies that aim to underpin our claims about the beneficial effects of negative knowledge and about its added value.

The propositions advanced here have implications for the design of vocational and professional education programs. As stated, negative knowledge is expected to emerge from error episodes that are experienced and analyzed through reflection. Therefore, to support such learning to happen in work contexts, vocational education programs should give trainees opportunities to experience authentic, complex and challenging errors, to learn from them and to thus build up negative knowledge. An interesting approach in this respect is error management training (Keith and Frese 2005; Nordstrom et al. 1998). In this form of training, errors are encouraged and consciously utilized as an integral part of learning processes. Studies have shown the beneficial effects of this form of training in terms of actual performance after the training, especially when complex tasks are to be developed (Keith and Frese 2005). The concept of negative knowledge and the error management training approach could be fruitfully combined in different ways. On the practical level, trainees could be asked to keep negative knowledge-diaries where they write down lessons learned from error episodes. Putting together these "what not to do"-statements from a whole courses' diaries could result in a "guidebook to catastrophe", similar to the above-mentioned example from the Nepalese hospital. On the scientific level, 
negative knowledge could add to the theoretic foundation of error management training and provide an additional possibility to empirically assess its effects.

In suggesting possibilities for how the general concept of negative knowledge can be fruitfully related to existing concepts and their empirical investigation, we draw on the general assertion of other theorists that negativity is a constitutive and central element of learning processes in general (English 2005). Thus, we hope to encourage the consideration of the idea in wider educational discourses.

Acknowledgements This work was supported by the Deutsche Forschungsgemeinschaft (German Research Foundation), grant Gr 1384/11-1. We thank Jaana Parviainen (Tampere University, Finland), Stephen Billett (Griffith University, Australia) and the anonymous reviewers for their helpful comments.

\section{References}

Bandura, A. (1997). Self-efficacy: The exercise of control. New York: Freeman.

Bauer, J., \& Mulder, R. H. (2007). Modelling learning from errors in daily work. Learning in Health and Social Care, 6, 121-133.

Billett, S. (2001). Learning in the workplace: Strategies for effective practice. Sydney: Allen and Unwin.

Boshuizen, H. P. A., Bromme, R., \& Gruber, H. (Eds.). (2004). Professional learning: Gaps and transitions on the way from novice to expert. Dordrecht: Kluwer.

Boud, D. J. (1999). Experience and learning: Reflection at work. Deakin: Deakin University, Faculty of Education.

Brewer, W. F. (1986). What is autobiographical memory? In D. Rubin (Ed.) Autobiographical memory (pp. 25-49). New York: Cambridge University Press.

Brodbeck, F. C., Zapf, D., Prümper, J., \& Frese, M. (1992). Error handling in office work with computers: A field study. Journal of Occupational and Organizational Psychology, 60, 303-317.

Crovitz, H. F., \& Schiffman, H. (1974). Frequency of episodic memories as a function of their age. Bulletin of the Psychonomic Society, 4, 517-518.

Edmondson, A. C. (1996). Learning from mistakes is easier said than done: Group and organizational influences on the detection and correction of human error. Journal of Applied Behavioral Science, 32, 5-28.

Ellis, S., \& Davidi, I. (2005). After-event reviews: Drawing lessons from successful and failed experience. Journal of Applied Psychology, 90, 857-871.

Ellström, P.-E. (2006). The meaning and role of reflection in informal learning at work. In D. J. Boud, P. Cressey, \& P. Docherty (Eds.) Productive reflection at work (pp. 43-53). London: Routledge.

English, A. (2005). Negativity and the new in John Dewey's theory of learning and democracy. Toward a renewed look at learning cultures. Zeitschrift für Erziehungswissenschaft, 1, 28-37.

Eraut, M. (1994). Developing professional knowledge and competence. London: Routledge Falmer.

Ericsson, K. A., Charness, N., Feltovich, P. J., \& Hoffman, R. R. (Eds.). (2006). Handbook on expertise and expert performance. Cambridge: Cambridge University Press.

Fahrenberg, J., \& Myrtek, M. (Eds.). (1996). Ambulatory assessment: Computer-assisted psychological and psychophysiological methods in monitoring and field studies. Seattle: Hogrefe and Huber.

Fenwick, T. (2006). Toward enriched conceptions of work learning: Participation, expansion, and translation among individuals with/in activity. Human Resource Development Review, 5, 285-302.

Flanagan, J. C. (1954). The critical incident technique. Psychological Bulletin, 51, 327-358.

Flavell, J. (1979). Metacognition and cognitive monitoring: A new area of cognitive developmental inquiry. American Psychologist, 34, 906-911.

Frese, M. (1991). Error management or error prevention: Two strategies to deal with errors in software design. In H.-J. Bullinger (Ed.) Human aspects in computing: Design and use of interactive systems and work with terminals (pp. 776-782). Amsterdam: Elsevier.

Frese, M. (1995). Error management in training: Conceptual and empirical results. In C. Zucchermaglio, S. Bagnara, \& S. Stucky (Eds.) Organizational learning and technological change (pp. 112-124). Berlin: Springer.

Frese, M., \& Zapf, D. (1994). Action as the core of work psychology: A German approach. In H. C. Triandis, M. D. Dunette, \& L. M. Hough (Eds.) Handbook of industrial and organizational psychology (2nd ed., vol. 4, pp. 271-340). Palo Alto: Consulting Psychologists. 
Furnham, A., \& Ribchester, T. (1995). Tolerance of ambiguity: A review of the concept, its measurement and applications. Current Psychology, 14, 179-199.

Gruber, H., \& Palonen, T. (Eds.). (2007). Learning in the workplace-new developments. Turku: Finnish Educational Research Association (FERA).

Harteis, C., Bauer, J., \& Haltia, P. (2007). Learning from errors in the workplace-insights from two studies in Germany and Finland. In H. Gruber, \& T. Palonen (Eds.) Learning in the workplace-new developments (pp. 119-138). Turku: Finnish Educational Research Association (FERA).

Heid, H. (1996). Erfordernis und Problematik einer Unterscheidung zwischen Verhalten und Verhaltensdisposition [The need and difficulty of a distinction between behavior and disposition of behavior]. In K. Beck, W. Müller, T. Deißinger, \& M. Zimmermann (Eds.) Berufserziehung im Umbruch (pp. 7985). Weinheim: Deutscher Studien.

Heid, H. (1999). Autorität-über die Verwandlung von Fehlern in Verfehlungen [Authority-the transformation of errors into misconducts]. In W. Althof (Ed.) Fehlerwelten. Vom Fehlermachen und Lernen aus Fehlern (pp. 129-136). Opladen: Leske + Budrich.

Helmreich, R. L., \& Merritt, A. C. (2000). Safety and error management: The role of crew resource management. In B. J. Hayward, \& A. R. Lowe (Eds.) Aviation resource management (pp. 107-119). Aldershot: Ashgate.

Hoffman, R. R., \& Lintern, G. (2006). Eliciting and representing the knowledge of experts. In K. A. Ericsson, N. Charness, P. J. Feltovich, \& R. R. Hoffman (Eds.) Handbook on expertise and expert performance (pp. 203-222). Cambridge: Cambridge University Press.

Keith, N., \& Frese, M. (2005). Self-regulation in error-management training: Emotion control and metacognition as mediators of performance effects. Journal of Applied Psychology, 90, 677-691.

Kolb, D. A. (1984). Experiential learning. Englewood Cliffs: Prentice-Hall.

Kolodner, J. (1983). Towards an understanding of the role of experience in the evolution from novice to expert. International Journal of Man-Machine Studies, 19, 497-518.

Kolodner, J. (1993). Case-based reasoning. San Mateo: Kaufman.

Lave, J., \& Wenger, E. (1991). Situated learning. Legitimate peripheral participation. Cambridge: Cambridge University Press.

Minsky, M. (1994). Negative expertise. International Journal of Expert Systems, 7(1), 13-19.

Morris, M. W., \& Moore, P. C. (2000). The lessons we (don't) learn: Counterfactual thinking and organizational accountability after a close call. Administrative Science Quarterly, 45, 737-765.

Nordstrom, C. R., Wendland, D., \& Williams, K. B. (1998). "To err is human": An examination of the effectiveness of error management training. Journal of Business and Psychology, 12, 269-282.

Oser, F., \& Spychiger, M. (2005). Lernen ist schmerzhaft. Zur Theorie des negativen Wissens und zur Praxis der Fehlerkultur [Learning is painful. On the theory of negative knowledge and the practice of error culture]. Weinheim: Beltz.

Parviainen, J., \& Eriksson, M. (2006). Negative knowledge, expertise and organisations. International Journal of Management Concepts and Philosophy, 2, 140-153.

Perrow, C. (1984). Normal accidents: Living with high risk technologies. Princeton: Princeton University Press.

Pintrich, P. R. (2002). The role of metacognitive knowledge in learning, teaching and assessing. Theory Into Practice, 41, 219-225.

Reason, J. T. (1990). Human error. Cambridge: Cambridge University Press.

Rochlin, G. I. (1999). Safe operation as a social construct. Ergonomics, 42, 1549-1560.

Rybowiak, V., Garst, H., Frese, M., \& Batinic, B. (1999). Error Orientation Questionnaire (EOQ): Reliability, validity and different language equivalence. Journal of Organizational Behavior, 20, 527-547.

Schank, R. C. (1999). Dynamic memory revisited. Cambridge: Cambridge University Press.

Schmidt, H. G., \& Boshuizen, H. P. A. (1993). On acquiring expertise in medicine. Educational Psychology Review, 5, 205-221.

Smith, P. J. (2003). Workplace learning and flexible delivery. Review of Educational Research, 73, 53-88.

van Dyck, C., Frese, M., Baer, M., \& Sonnentag, S. (2005). Organizational error management culture and its impact on performance: A two-study replication. Journal of Applied Psychology, 90, 1228-1240.

van Woerkom, M. (2003). Critical reflection at work. Bridging individual and organisational learning. Enschede: PrintPartners.

von Glasersfeld, E. (1991). A constructivist's view of learning and teaching. In R. Duit, F. Goldberg, \& H. Niedderer (Eds.) Research in physics learning: Theoretical issues and empirical studies (pp. 29-39). Kiel: IPN.

Wood, R. H., \& Sweggins, R. W. (2006). Aircraft accident investigation (2nd ed.). Casper: Endeavour. 